International Journal of Pure and Applied Mathematics

Volume 92 No. 5 2014, 669-690

ISSN: 1311-8080 (printed version); ISSN: 1314-3395 (on-line version)

url: http://www.ijpam.eu

doi: http://dx.doi.org/10.12732/ijpam.v92i5.4

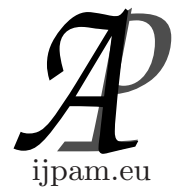

\title{
SOME CONFIGURATIONS OF LINES WHOSE IDEALS ARE LIFTINGS OF MONOMIAL IDEALS
}

\author{
Margherita Guida ${ }^{1}$, Ferruccio Orecchia ${ }^{1}$, Luciana Ramella ${ }^{2}$ \\ ${ }^{1}$ Dipartimento di Matematica e Appl. \\ Università di Napoli "Federico II", \\ Via Cintia, 80126 Napoli, ITALY \\ ${ }^{2}$ Dipartimento di Matematica \\ Università di Genova \\ v. Dodecaneso 35, 16146 Genova, ITALY
}

\begin{abstract}
In this paper we consider some configurations of lines whose ideals are generated by a product of linear forms. We show that in general these ideals are 1-lifting or pseudo 1-lifting of monomial ideals. This proves also that these ideals are Arithmetically Cohen Macaulay.
\end{abstract}

AMS Subject Classification: 14Q05

Key Words: lifting, lines

\section{Introduction}

The topic of ideals $I$ generated by products of linear forms has raised interest in many authors (see for example [1],[9],[12]). It is clear that the ideals $I$ are union of linear varieties, but in general a union of linear varieties is not generated by products of linear forms (see [1], Proposition 5.7). It is also clear, by definition, that lifting and pseudo 1-lifting of monomial ideals (as defined by Migliore and Nagel [11]) are generated by products of linear forms and Cohen Macaulay but the converse is not true (see Example 4.13). In [2] the third author with Cioffi and Marinari studied geometric properties of union of linear varieties defined by liftings and pseudo 1-liftings of monomial ideals. Furthermore in [7] the first two author introduced configurations of lines, called

Received: January 28, 2014

(c) 2014 Academic Publications, Ltd.

$\S_{\text {Correspondence author }}$ url: www.acadpubl.eu 
grids, which are generated by products of linear forms. It is natural to ask if the grids are 1-lifting of monomial ideals. In this paper we show that complete grids are 1-liftings of monomial ideals. We show also that incomplete grids can be not pseudo 1-lifting. Moreover we prove that the complete grids of fat lines (introduced in [8]) are 1-lifting of monomial ideals (see Theorem 3.5) and then we prove (Corollary 3.6) that these configurations of lines are A.C.M. in $\mathbb{P}^{n}$, thus generalizing this result proved in $[8]$ in $\mathbb{P}^{3}$. Finally we describe some more general configurations of lines in $\mathbb{P}^{3}$ (see Definition 4.2) that are A.C.M. (Proposition 4.5). The Cohen Macaulay property is proved by studying the behaviour of their hyperplane section as in [7]. These configurations can be 1-lifting, pseudo 1-lifting, and not pseudo 1-lifting.

\section{Lifting Monomial Ideals}

We recall and illustrate same definitions and results given in [11] and [2] about liftings and pseudoliftings of monomial ideals defining configurations of linear projective varieties.

From now on $K$ will be a field with characteristic 0 , for $n \in \mathbb{N}^{*}$ and $t \in$ $\mathbb{N} x_{1}, \ldots, x_{n}, u_{1}, \ldots, u_{t}$ will be indeterminates, $S:=K\left[x_{1}, \ldots, x_{n}\right]$ and $R:=$ $K\left[x_{1}, \ldots, x_{n}\right.$, $\left.u_{1}, \ldots, u_{t}\right]$. For $t=1$ we put $u_{1}=x_{0}$.

Let $A=\left(L_{j, i}\right)_{1 \leq j \leq n, 1 \leq i \leq r}$ be a matrix whose entries $L_{j, i}$ are linear forms in $R$ and consider the following conditions (see Proposition 1.7 of [2]):

Condition $(\alpha)$. The $n$ linear forms $L_{1, i_{1}}, \ldots, L_{n, i_{n}}$ are linearly independent for every $1 \leq i_{1}, \ldots, i_{n} \leq r$.

Condition $\left(\alpha^{\prime}\right)$. The vector spaces $<L_{1, i_{1}}, \ldots, L_{n, i_{n}}>$ are $n$ dimensional and pairwise distinct.

Definition 1.1. Consider an $n \times r$ matrix $A$ of linear forms $L_{j, i} \in R$ satisfying Condition $(\alpha)$. To a monomial $m=x_{1}^{\alpha_{1}} \ldots x_{n}^{\alpha_{n}} \in S$, with $\alpha_{k} \leq r$ for every $1 \leq k \leq r$, we associate the homogeneous polynomial

$$
\tilde{m}=\prod_{j=1}^{n}\left(\prod_{i=1}^{\alpha_{j}} L_{j, i}\right) \in R .
$$

To a monomial ideal $J=\left(m_{1}, \ldots, m_{s}\right) \subset S$, where $\left\{m_{1}, \ldots, m_{s}\right\}$ is the minimal system of monic monomial-generators of $J$ and the largest power of $x_{j}$ 
occurring as a factor of any of the minimal generators of $J$ is lower or equal to $r$, we associate the homogeneous ideal $I=\left(\tilde{m}_{1}, \ldots, \tilde{m}_{s}\right) \subset R$.

Then $I$ is called a pseudo-t-lifting of $J$ and $A$ is called a pseudo-t-lifting matrix ([11] Definition 2.22).

If the linear forms $L_{j, i}$ of a pseudo-t-lifting matrix $A$ actually belong to $K\left[x_{j}, u_{1}\right.$,

$\left.\ldots, u_{t}\right] \subset R$ and $x_{j}$ has non-zero coefficient, then the pseudo-t-lifting $I$ of a monomial ideal $J$ via $A$ is called a $t$-lifting of $J$. In fact $R /\left(I, u_{1}, \ldots, u_{t}\right) \cong S / J$ (see [11]).

Now we recall some properties about monomial ideals $J$ and their order ideals $\mathcal{N}(J)$. The geometric representation of $\mathcal{N}(J)$ given in [10] was used in [2] to describe the irreducible components of the projective scheme $V(I) \subseteq \mathbb{P}_{K}^{n+t-1}$ defined by a pseudo-t-lifting $I$ of $J$.

Definition 1.2. A set $\mathcal{N} \subseteq \mathbb{N}^{n}$ is an order ideal if and only if $\left(a_{1}, \ldots, a_{n}\right) \in$ $\mathcal{N}$ and $\left(b_{1}, \ldots, b_{n}\right) \in \mathbb{N}^{n}$ with $b_{j} \leq a_{j}$ for every $1 \leq j \leq n$ implies $\left(b_{1}, \ldots, b_{n}\right) \in$ $\mathcal{N}$.

For every monomial ideal $J \subset S$ the order ideal $\mathcal{N}(J):=\left\{\left(a_{1}, \ldots, a_{n}\right) \in\right.$ $\left.\mathbb{N}^{n}: x_{1}^{a_{1}} \cdots x_{n}^{a_{n}} \notin J\right\}$ is called order ideal of $J$.

Proposition 1.3. (Proposition 2.5 of [2]) Let $V(I) \subseteq \mathbb{P}_{K}^{n+t-1}$ be the projective scheme defined by a pseudo-t-lifting $I \subset R$ of a monomial ideal $J \subset S$ induced by an $n \times r$ matrix $A=\left(L_{j, i}\right)$ whose entries are linear forms satisfying Condition ( $\alpha)$. Then we have:

(1) $\left(L_{j_{1}, i_{1}}, \ldots, L_{j_{k}, i_{k}}\right)$ (with $j_{1}, \ldots, j_{k}$ distinct) gives an irreducible component of $V(I)$ iff $\left\{\left(a_{1}, \ldots, a_{n}\right) \in \mathbb{N}^{n}: a_{j_{1}}=i_{1}-1, \ldots, a_{j_{k}}=i_{k}-1\right\}$ is an irreducible component of $\mathcal{N}(J)$ (here $\mathbb{N}^{n}$ is considered a subspace of $\mathbb{A}_{\mathbb{Q}}^{n}$ with the Zariski topology).

(2) If Condition $\left(\alpha^{\prime}\right)$ holds, then $V(I)$ is reduced and there is a bijection between the irreducible components of $V(I)$ and the ones of $\mathcal{N}(J)$.

For pseudo-1-liftings we put $u_{1}=x_{0}$ and $R=K\left[x_{0}, x_{1}, \ldots, x_{n}\right]$. If $I$ is an ideal obtained by a pseudo-1-lifting of a monomial ideal of $S$, then we denote by $V_{a}(I)$ the intersection of $V(I) \subset \mathbb{P}_{K}^{n}$ with the open affine set $x_{0} \neq 0$.

If we consider a monomial ideal $J \subset S$ and the 1-lifting matrix $A$ with entries $L_{j, i}=x_{j}-(i-1) x_{0}, j=1, \ldots, n$, then the order ideal $\mathcal{N}(J) \subset \mathbb{N}^{n}$ of $J$ is $V_{a}(I) \cap \mathbb{N}^{n}$, where $I$ is the 1-lifting of $J$ via $A$.

The pseudo-1-liftings of unmixed monomial ideals $J \subset S$ with $\operatorname{dim}(S / J)=1$ give examples of A.C.M. configurations of lines. We can prove the following proposition by means of minimal resolutions as in [11]. 
Proposition 1.4. Let $J \subset S$ be an unmixed monomial ideal with $\operatorname{dim}(S / J)$ $=1$ and $I \subset R$ be a pseudo-1-lifting of $J$. Then $R / I$ is C.M..

Proof. The ring $S / J$ is C.M. because the monomial ideal $J$ is unmixed and $\operatorname{dim} S / J=1$. Moreover a monomial ideal $J$ and a pseudo- $t$-lifting $I$ of $J$ have the same Betti numbers (see e.g. [11]), then $S / J$ e $R / I$ have the same projective dimension. By using the Auslander - Buchsbaum formula in the graduate case:

$$
\operatorname{pd}_{R}(M)=\operatorname{depth}(\mathfrak{m}, R)-\operatorname{depth}(\mathfrak{m}, M)
$$

where $R$ is a graduate ring, $\mathfrak{m}$ the irrilevant maximal ideal, $M$ an $R$-module ([3]), we can prove as in [11] Corollary 2.10 that $R / I$ is C.M. if and only if $S / J$ is C.M..

Example 1.5. Consider in $S=K\left[x_{1}, x_{2}\right]$ the ideals $J=\left(x_{1}^{2}, x_{2}^{2}\right)$ (see Figure 1) and $J^{\prime}=\left(x_{1}^{2} x_{2}^{2}\right)$ (see Figure 2).

Let $A_{1}$ be the 1-lifting matrix satisfying Condition $\left(\alpha^{\prime}\right)$

$$
A_{1}=\left(\begin{array}{cc}
x_{1} & x_{1}-x_{0} \\
x_{2} & x_{2}-x_{0}
\end{array}\right)
$$

Then the 1-lifting of $J$ via $A_{1}$ is the ideal $I_{1}=\left(x_{1}\left(x_{1}-x_{0}\right), x_{2}\left(x_{2}-x_{0}\right)\right)=$ $\left(x_{1}, x_{2}\right) \cap\left(x_{1}, x_{2}-x_{0}\right) \cap\left(x_{1}-x_{0}, x_{2}\right) \cap\left(x_{1}-x_{0}, x_{2}-x_{0}\right)$ defining in $\mathbb{P}_{K}^{2}$ the set of points $\{(1,0,0),(1,0,1),(1,1,0),(1,1,1)\}$. While the 1 -lifting of $J^{\prime}$ via $A_{1}$ is $I_{1}^{\prime}=\left(x_{1}\left(x_{1}-x_{0}\right) x_{2}\left(x_{2}-x_{0}\right)\right)$ defining four lines (see Figure 3 ).

If $A_{2}$ is the 1-lifting matrix not satisfying Condition $\left(\alpha^{\prime}\right)$

$$
A_{2}=\left(\begin{array}{cc}
x_{1}-x_{0} & 2 x_{1}-2 x_{0} \\
x_{2} & x_{2}-x_{0}
\end{array}\right),
$$

then the 1-lifting of $J$ via $A_{2}$ is the ideal $I_{2}=\left(\left(x_{1}-x_{0}\right)^{2}, x_{2}\left(x_{2}-x_{0}\right)\right)=$ $\left(\left(x_{1}-x_{0}\right)^{2}, x_{2}\right) \cap\left(\left(x_{1}-x_{0}\right)^{2}, x_{2}-x_{0}\right)$ defining two double points. While the 1-lifting of $J^{\prime}$ via $A_{2}$ is $I_{2}^{\prime}=\left(\left(x_{1}-x_{0}\right)^{2} x_{2}\left(x_{2}-x_{0}\right)\right)$ defining two simple lines and a double line (see Figure 4 ).

Now consider the pseudo-1-lifting matrix $A_{3}$ satisfying Condition $\left(\alpha^{\prime}\right)$

$$
A_{3}=\left(\begin{array}{cc}
x_{1}+x_{2}+x_{0} & x_{1}-x_{2}-x_{0} \\
3 x_{1}-x_{2}+3 x_{0} & 2 x_{1}+x_{2}-2 x_{0}
\end{array}\right) .
$$

Then the pseudo-1-lifting of $J$ via $A_{3}$ is the ideal $I_{3}=\left(\left(x_{1}+x_{2}+x_{0}\right)\left(x_{1}-x_{2}-\right.\right.$ $\left.\left.x_{0}\right),\left(3 x_{1}-x_{2}+3 x_{0}\right)\left(2 x_{1}+x_{2}-2 x_{0}\right)\right)=\left(x_{1}+x_{2}+x_{0}, 3 x_{1}-x_{2}+3 x_{0}\right) \cap\left(x_{1}+\right.$ $\left.x_{2}+x_{0}, 2 x_{1}+x_{2}-2 x_{0}\right) \cap\left(x_{1}-x_{2}-x_{0}, 3 x_{1}-x_{2}+3 x_{0}\right) \cap\left(x_{1}-x_{2}-x_{0}, 2 x_{1}+x_{2}-\right.$ 
$\Delta$

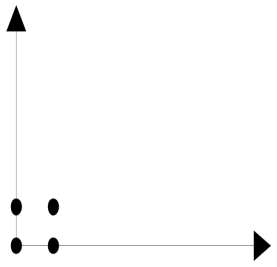

Figure 1: The order ideal in $\mathbb{N}^{2}$ of $J=$ $\left(x_{1}^{2}, x_{2}^{2}\right)$.

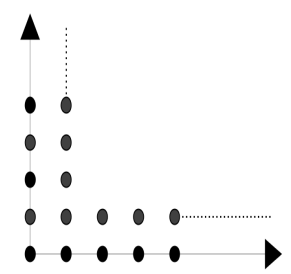

Figure 2: The order ideal in $\mathbb{N}^{2}$ of $J^{\prime}=$ $\left(x_{1}^{2} x_{2}^{2}\right)$.

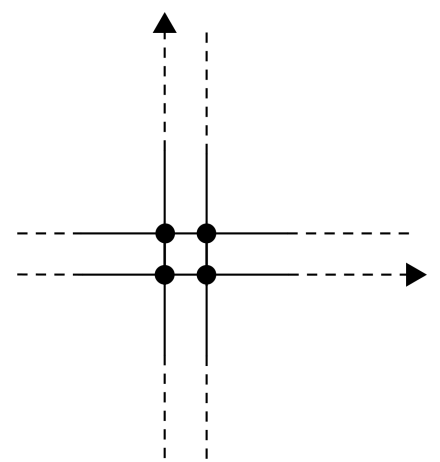

Figure 3: The four points $V_{a}\left(I_{1}\right)$ and the four lines $V_{a}\left(I_{1}^{\prime}\right)$ in $\mathbb{A}_{K}^{2}$ given by a reduced 1-lifting.

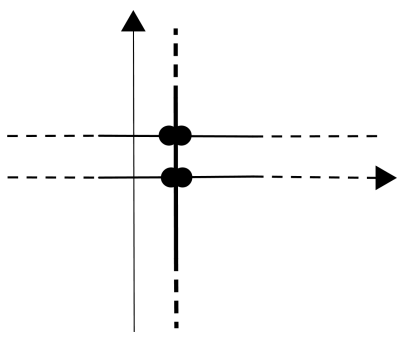

Figure 4: $V_{a}\left(I_{2}\right) \subset \mathbb{A}_{K}^{2}$ consisting of two double points and $V_{a}\left(I_{2}^{\prime}\right) \subset$ $\mathbb{A}_{K}^{2}$ consisting of a double line with two simple lines are non-reduced 1liftings

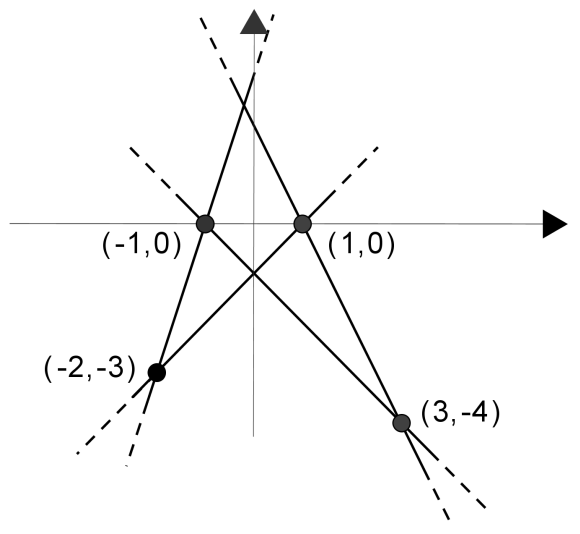

Figure 5: The four points $V_{a}\left(I_{3}\right) \subset$ $\mathbb{A}_{K}^{2}$ and the four lines $V_{a}\left(I_{3}^{\prime}\right) \subset \mathbb{A}_{K}^{2}$ given by a reduced pseudo-1-lifting that is not an 1-lifting.

$\left.\left.2 x_{0}\right)\right)$ defining in $\mathbb{P}_{K}^{2}$ the set of points $\{(1,-1,0),(1,3,-4),(1,-2,-3),(1,1,0)\}$. While the pseudo-1-lifting of $J^{\prime}$ via $A_{3}$ is $I_{3}^{\prime}=\left(\left(x_{1}+x_{2}+x_{0}\right)\left(x_{1}-x_{2}-\right.\right.$ $\left.\left.x_{0}\right)\left(3 x_{1}-x_{2}+3 x_{0}\right)\left(2 x_{1}+x_{2}-2 x_{0}\right)\right)$ defining four lines (see Figure 5$)$. Note that $\left(I_{3}, x_{0}\right) /\left(x_{0}\right) \neq J$ and $\left(I_{3}^{\prime}, x_{0}\right) /\left(x_{0}\right) \neq J^{\prime}$. 


\section{The Complete Grids of Lines are Liftings}

Now we recall definitions given in [7] about lattice of points and complete grids of lines.

Definition 2.1. Let $\left\{a_{11}, \ldots, a_{1 \ell_{1}}\right\}, \ldots,\left\{a_{n 1}, \ldots, a_{n \ell_{n}}\right\}$ be $n$ finite subsets of elements of $K$. The finite set

$$
X:=\left\{\left(1, a_{1 i_{1}}, \ldots, a_{n i_{n}}\right): i_{k}=1, \ldots, \ell_{k}, k=1, \cdots, n\right\} \subset \mathbb{P}_{K}^{n}
$$

consisting of $\ell_{1} \ell_{2} \cdots \ell_{n}$ points, is called a lattice of type $\left(\ell_{1}, \ldots, \ell_{n}\right)$.

If $\ell_{1}=\ldots=\ell_{n}=r$, then $X$ is called a cubic lattice of type $r$.

Remark 2.2. Consider, for $k=1, \ldots n$, the points $P_{\infty, k}=\left(\xi_{0 k}, \xi_{1 k}, \ldots, \xi_{n k}\right)$ $\in \mathbb{P}_{K}^{n}$, where $\xi_{i k}=0$ for $i \neq k$ and $\xi_{k k}=1$. Note that for every point $P=\left(1, a_{1 i_{1}}, \ldots, a_{n i_{n}}\right)$ of a lattice $X$ of type $\left(\ell_{1}, \ldots, \ell_{n}\right)$, the line $r_{P, k}$ jointing the points $P$ and $P_{\infty, k}$ contains $\ell_{k}$ points of $X$ whose coordinates are $\left(1, a_{1 i_{1}}, \ldots, a_{k i_{k}}, \ldots, a_{n i_{n}}\right)$, with $1 \leq i_{k} \leq \ell_{k}$.

Definition 2.3. Let $X$ be a lattice of type $\left(\ell_{1}, \ldots, \ell_{n}\right)$ of points of $\mathbb{P}_{K}^{n}$. The finite set

$$
Y:=\left\{r_{P, k}: P \in X, 1 \leq k \leq n\right\}
$$

of the $\left(\ell_{2} \ell_{3} \cdots \ell_{n}+\cdots+\ell_{1} \cdots \ell_{j-1} \ell_{j+1} \cdots \ell_{n}+\cdots+\ell_{1} \cdots \ell_{n-1}\right)$ lines $r_{P, k}$ jointing a point $P$ of $X$ and one of the point $P_{\infty, k}$ (see above Remark) is called a complete grid of type $\left(\ell_{1}, \ldots, \ell_{n}\right)$ with basis $X$.

A grid line is a line of a complete grid.

Example 2.4. In Figure 3 the four points $V_{a}\left(I_{1}\right)$ are a lattice and the four lines $V_{a}\left(I_{1}^{\prime}\right)$ are a grid of lines of type $(2,2)$. While in Figure 5 the four points $V_{a}\left(I_{3}\right)$ are not a lattice and the four lines $V_{a}\left(I_{3}^{\prime}\right)$ do not form a grid of lines.

The ideals defining lattices of points and complete grids of lines are calculated in [7]. Now we prove that these ideals are 1-liftings of monomial ideals.

Lemma 2.5. Let $X=\left\{\left(1, a_{1 i_{1}}, \ldots, a_{n i_{n}}\right): 1 \leq k \leq n, 1 \leq i_{k} \leq r\right\}$ be a cubic lattice of points in $\mathbb{P}_{K}^{n}$ of type $r$. Then $\Lambda=\left(x_{j}-a_{j i} x_{0}\right)_{1 \leq j \leq n, 1 \leq i \leq r}$ is an 1- lifting matrix satisfying Condition $\left(\alpha^{\prime}\right)$.

Proof. The $n$-dimensional vector spaces $<x_{1}-a_{1 i_{1}} x_{0}, \ldots, x_{n}-a_{n, i_{n}} x_{0}>$ are distinct because the points of the lattice $X$ are distinct. So Condition $\left(\alpha^{\prime}\right)$ is verified by the matrix $\Lambda$.

Theorem 2.6. Let $X$ be a lattice of type $\left(\ell_{1}, \ldots, \ell_{n}\right)$ of points of $\mathbb{P}_{K}^{n}$ and put $r=\max \left\{\ell_{1}, \ldots, \ell_{n}\right\}$. Then the lattice $X$ can be completed to a cubic 
lattice $\bar{X}$ of type $r$. Consider the 1-lifting matrix $\Lambda$ determined by $\bar{X}$ as in Lemma 2.5. Then the ideal $I_{X}$ defining $X$ is the 1-lifting of the monomial ideal $J=\left(x_{1}^{\ell_{1}}, \ldots, x_{n}^{\ell_{n}}\right)$ via the matrix $\Lambda$.

Moreover, if $Y$ is a complete grid of type $\left(\ell_{1}, \ldots, \ell_{n}\right)$ with basis the lattice $X$ of points, then the defining ideal $I_{Y}$ of $Y$ is the 1-lifting via the matrix $\Lambda$ of the monomial ideal $J^{\prime}=\cap_{k=1}^{n}\left(x_{1}^{\ell_{1}}, \ldots, x_{k}^{\hat{\ell}_{k}}, \ldots, x_{n}^{\ell_{n}}\right)$, which is generated by $\left\{x_{j}^{\ell_{j}} x_{k}^{\ell_{k}}: 1 \leq j<k \leq n\right\}$.

Proof. Put $L_{j, i}:=x_{j}-a_{j i} x_{0}, j=1, \ldots, n, i=1, \ldots, r$, with $r \geq \ell_{j}$ for every $j$. From [7] Lemma 3(see also [4]) the ideal defining $X$ is $I_{X}=$ $\left(\prod_{i=1}^{\ell_{1}} L_{1, i}, \ldots, \prod_{i=1}^{\ell_{n}} L_{n, i}\right)$, that is the 1-lifting of the monomial ideal $J$ induced by the 1-lifting matrix $\Lambda$.

If we prove that the ideal $I_{Y}$ is generated by $\left\{\left(\prod_{i=1}^{\ell_{j}} L_{j, i}\right)\left(\prod_{i=1}^{\ell_{k}} L_{k, i}\right): j<\right.$ $k, j, k \in\{1, \ldots, n\}\}$ then $I_{Y}$ is the 1-lifting of the monomial ideal $J^{\prime}$ induced by the matrix $\Lambda$, as claimed. By the definition of complete grid of type $\left(\ell_{1}, \ldots, \ell_{n}\right)$ we have that $I_{Y}$ is:

$$
\begin{aligned}
I_{Y}=\bigcap_{i=1}^{\ell_{2}}\left(L_{2, i}, L_{3,1}, \ldots, L_{n, 1}\right) \bigcap \ldots \\
\\
\bigcap_{i=1}^{\ell_{2}}\left(L_{2, i}, L_{3, \ell_{3}}, \ldots, L_{n, \ell_{n}}\right) \bigcap_{i=1}^{\ell_{1}}\left(L_{1, i}, L_{3,1}, \ldots, L_{n, 1}\right) \\
\quad \bigcap \ldots \bigcap_{i=1}^{\ell_{1}}\left(L_{1, i}, L_{3, \ell_{3}}, \ldots, L_{n, \ell_{n}}\right) \bigcap \ldots \bigcap_{i=1}^{\ell_{n-1}}\left(L_{1, \ell_{1}}, \ldots, L_{n-2, \ell_{n-2}}, L_{n-1, i}\right) .
\end{aligned}
$$

By applying the Lemma 7 of [7] we obtain that:

$$
\begin{gathered}
I_{Y}=\left(\prod_{i=1}^{\ell_{2}} L_{2, i}, L_{3,1}, \ldots, L_{n, 1}\right) \bigcap \ldots \\
\bigcap\left(\prod_{i=1}^{\ell_{2}} L_{2, i}, L_{3, \ell_{3}}, \ldots, L_{n, \ell_{n}}\right) \bigcap\left(\prod_{i=1}^{\ell_{1}} L_{1, i}, L_{3,1}, \ldots, L_{n, 1}\right) \\
\bigcap \ldots \bigcap\left(\prod_{i=1}^{\ell_{1}} L_{1, i}, L_{3, \ell_{3}}, \ldots, L_{n, \ell_{n}}\right) \bigcap \ldots \bigcap\left(L_{1, \ell_{1}}, \ldots, L_{n-2, \ell_{n-2}}, \prod_{i=1}^{\ell_{n-1}} L_{n-1, i}\right) .
\end{gathered}
$$

By the modular law we have

$$
I_{Y}=\left(\prod_{i=1}^{\ell_{1}} L_{1, i} \prod_{j=1}^{\ell_{2}} L_{2, j}, \ldots, \prod_{i=1}^{\ell_{n-1}} L_{n-1, i} \prod_{j=1}^{\ell_{n}} L_{n, j}\right) .
$$


Remark 2.7. By Proposition 1.3, the points of a lattice (resp. the lines of a complete grid) of type $\left(\ell_{1}, \ldots, \ell_{n}\right)$ in $\mathbb{P}_{K}^{n}$ can be "read" in $\mathbb{N}^{n}$ looking at the order ideal of the artinian monomial ideal $J=\left(x_{1}^{\ell_{1}}, \cdots, x_{n}^{\ell_{n}}\right)$ (resp. of the unmixed monomial ideal $J^{\prime}=\left(x_{1}^{\ell_{1}} x_{2}^{\ell_{2}}, x_{1}^{\ell_{1}} x_{3}^{\ell_{3}}, \ldots, x_{n-1}^{\ell_{n}-1} x_{n}^{\ell_{n}}\right)$. Note that, by using results of [2], we can prove that every 1-lifting of $J$ (resp. $J^{\prime}$ ) via an 1-lifting matrix satisfying Condition $\left(\alpha^{\prime}\right)$ is a lattice of points (resp. a complete grid of lines).

Definition 2.8. We say that $J=\left(x_{1}^{\ell_{1}}, \cdots, x_{n}^{\ell_{n}}\right)$ is the monomial ideal giving the lattices of points of type $\left(\ell_{1}, \ldots, \ell_{n}\right)$ and $J^{\prime}=\left(x_{1}^{\ell_{1}} x_{2}^{\ell_{2}}, x_{1}^{\ell_{1}} x_{3}^{\ell_{3}}, \ldots, x_{n-1}^{\ell_{n-1}} x_{n}^{\ell_{n}}\right)$ is the monomial ideal giving the complete grids of lines of type $\left(\ell_{1}, \ldots, \ell_{n}\right)$.

Example 2.9. A lattice of points and a complete grid of lines of type $(2,3,2)$. Consider in $S=K\left[x_{1}, x_{2}, x_{3}\right]$ the ideals $J=\left(x_{1}^{2}, x_{2}^{3}, x_{3}^{2}\right)$ and $J^{\prime}=$ $\left(x_{1}^{2} x_{2}^{3}, x_{1}^{2} x_{3}^{2}, x_{2}^{3} x_{3}^{2}\right)$.

Let $A$ be the 1-lifting matrix satisfying Condition $\left(\alpha^{\prime}\right)$

$$
A=\left(\begin{array}{ccc}
x_{1} & x_{1}-3 x_{0} & x_{1}+a_{1,3} x_{0} \\
x_{2}-x_{0} & x_{2}-4 x_{0} & x_{2}-6 x_{0} \\
x_{3}-2 x_{0} & x_{3}-5 x_{0} & x_{3}+a_{3,3} x_{0}
\end{array}\right)
$$

Then the 1-lifting of $J$ via $A$ is the ideal $I=\left(x_{1}\left(x_{1}-3 x_{0}\right),\left(x_{2}-x_{0}\right)\left(x_{2}-\right.\right.$ $\left.\left.4 x_{0}\right)\left(x_{2}-6 x_{0}\right),\left(x_{3}-2 x_{0}\right)\left(x_{3}-5 x_{0}\right)\right)$ defining in $\mathbb{P}_{K}^{3}$ the set $X$ of 12 points represented by Figure 6 in the open set $x_{0} \neq 0$. While the 1-lifting of $J^{\prime}$ via $A$ is $I^{\prime}=\left(x_{1}\left(x_{1}-3 x_{0}\right)\left(x_{2}-x_{0}\right)\left(x_{2}-4 x_{0}\right)\left(x_{2}-6 x_{0}\right), x_{1}\left(x_{1}-3 x_{0}\right)\left(x_{3}-2 x_{0}\right)\left(x_{3}-\right.\right.$ $\left.\left.5 x_{0}\right),\left(x_{2}-x_{0}\right)\left(x_{2}-4 x_{0}\right)\left(x_{2}-6 x_{0}\right)\left(x_{3}-2 x_{0}\right)\left(x_{3}-5 x_{0}\right)\right)$ defining a set $Y$ of 16 lines (Figure 7).

Note that $X$ is a lattice of points in $\mathbb{P}_{K}^{3}$ of type $(2,3,2)$ and $Y$ is a complete grid with basis $X$.

Definition 2.10. A subset $\mathcal{A}$ of grid lines of a complete grid $Y$ is called incomplete grid.

In [7], [5] particular types of incomplete grids are considered. Here we exhibit a class of incomplete grids related to monomial ideals.

Proposition 2.11. Let $J \subset S$ be a monomial unmixed ideal such that $\operatorname{dim}(S / J)=1$ and $n \geq 2$. Then any 1-lifting $I$ of $J$ induced by a matrix satisfying Condition $\left(\alpha^{\prime}\right)$ defines an incomplete grid $V=V(I) \subset \mathbb{P}_{K}^{n}$.

Proof. Let $I$ be a 1-lifting of $J$ induced by a matrix satisfying Condition $\left(\alpha^{\prime}\right)$. By definition, $J=\cap_{k=1}^{n} J_{k}$, where, for $k=1, \ldots, n, J_{k}$ is a monomial ideal whose minimal generators do not involve the indeterminate $x_{k}$, 


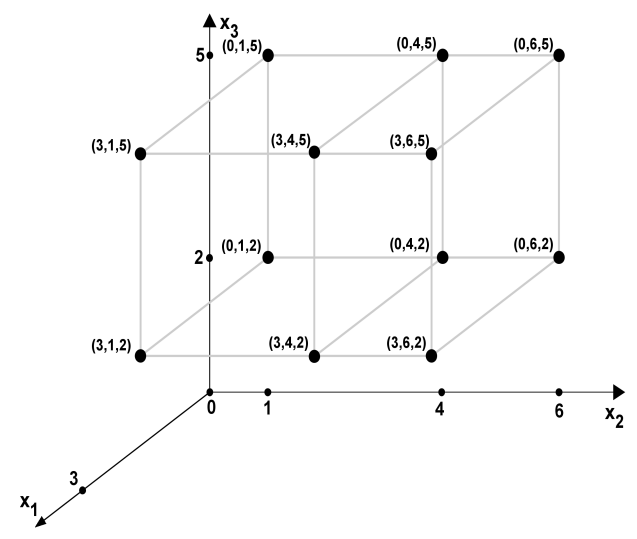

Figure 6: The lattice $X$ in $\mathbb{A}_{K}^{3}\left(x_{0} \neq\right.$ $0)$ of type $(2,3,2)$ consisting of 12 points given by the 1-lifting of $J=$ $\left(x_{1}^{2}, x_{2}^{3}, x_{3}^{2}\right)$ via the matrix $A$.

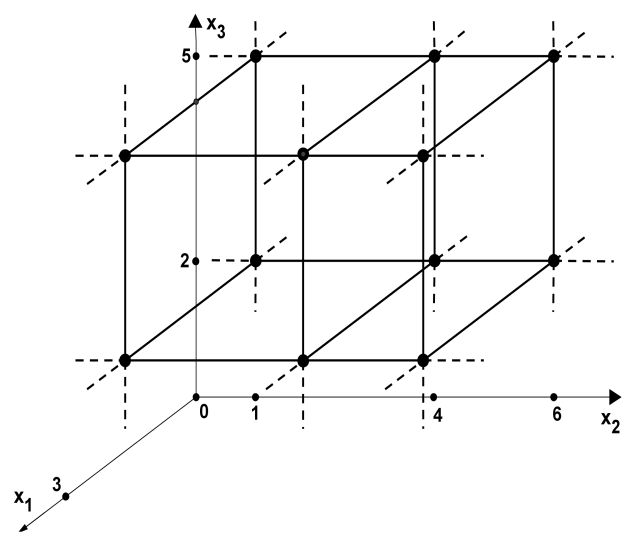

Figure 7: The trace in $\mathbb{A}_{K}^{3}\left(x_{0} \neq 0\right)$ of the complete grid $Y$ with basis $X$ given by the 1-lifting of $J^{\prime}=$ $\left(x_{1}^{2} x_{2}^{3}, x_{1}^{2} x_{3}^{2}, x_{2}^{3} x_{3}^{2}\right)$ via the matrix $A$.

i.e. $J_{k}$ is the extension of a monomial artinian ideal in the $n-1$ indeterminates $x_{1}, \ldots, \widehat{x_{k}}, \ldots, x_{n}$. Then, there exist positive integers $\ell_{1}, \ldots, \ell_{n}$ such that $\left(x_{1}^{\ell_{1}}, \ldots, x_{k}^{\ell_{k}}, \ldots, x_{n}^{\ell_{n}}\right) \subseteq J_{k}$ for every $k=1, \ldots, n$. Thus

$$
\begin{aligned}
\left(x_{1}^{\ell_{1}} x_{2}^{\ell_{2}}, x_{1}^{\ell_{1}} x_{3}^{\ell_{3}}, \ldots, x_{1}^{\ell_{1}} x_{n}^{\ell_{n}}, \ldots, x_{n-1}^{\ell_{n-1}} x_{n}^{\ell_{n}}\right)=\cap_{k=1}^{n}\left(x_{1}^{\ell_{1}}, \ldots, \widehat{x_{k}^{\ell_{k}}}, \ldots, x_{n}^{\ell_{n}}\right) & \\
& \subseteq \cap_{k=1}^{n} J_{k}=J .
\end{aligned}
$$

Hence, $V(I)$ is an incomplete grid in $\mathbb{P}_{K}^{n}$.

Example 2.12. Incomplete grid with 8 lines. Configuration defined by a 1-lifting of a monomial ideal. Consider an incomplete grid constructed from the edges of a cube (of the euclidean space $\mathbb{E}_{\mathbb{R}}^{3}$ ) corresponding to a complete grid of type $(2,2,2)$ by eliminating four lines parallel to same coordinate axis for example $y$. The projective closure of these 8 lines is given by the 1-lifting of the monomial ideal $J=\left(x_{2}^{2}, x_{1}^{2} x_{3}^{2}\right)$ induced by the matrix $A$ :

$$
A=\left(\begin{array}{ll}
x_{1} & x_{1}-x_{0} \\
x_{2} & x_{2}-x_{0} \\
x_{3} & x_{3}-x_{0}
\end{array}\right) .
$$

Remark 2.13. A complete grid of lines is A.C.M., this statement was proved in [7] by studying the algebraic intersection with a hyperplane. Note 
that the assertion follows also because a complete grid of lines is an 1-lifting (Proposition 2.6) of an unmixed monomial ideal satisfying the condition of Proposition 1.4.

Remark 2.14. In [7] Section 4, there are examples of incomplete grids of lines that are not A.C.M., so these configurations of lines are not liftings of monomial ideals.

Remark 2.15. Note that the configurations of lines given by the first author in [6] Theorem 3 are pseudo-1-liftings of the monomial ideals $J^{\prime}$ of Proposition 2.6 giving complete grids of lines (Definition 2.8). Also these configuration of lines are A.C.M., by Theorem 4 of [6] or Proposition 1.4 and [11].

Remark 2.16. In [5] the first author computes the minimal free resolution of a complete grid of $\mathbb{P}_{K}^{3}$ of type $(l, m, n)$ and poses a conjecture about the minimal free resolution of a complete grid of $\mathbb{P}_{K}^{n}$ of type $\left(l_{1}, \ldots, l_{n}\right)$.

\section{The $m$-Fat Complete Grids of Lines are Liftings}

Definition 3.1. Let $m_{1}, \ldots, m_{h}$ be positive integers, a fat lattice (resp. fat complete grid) of type $\left(\ell_{1}, \ldots, \ell_{n}\right)$ is the projective scheme

$$
\operatorname{Proj}\left(K\left[x_{0}, \ldots, x_{n}\right] /\left(\mathcal{P}_{1}^{m_{1}} \cap \cdots \cap \mathcal{P}_{h}^{m_{h}}\right),\right.
$$

whose support is a lattice of points (resp. a complete grid of lines) $V\left(\mathcal{P}_{1}\right) \cup$ $\cdots \cup V\left(\mathcal{P}_{h}\right)$ of type $\left(\ell_{1}, \ldots, \ell_{n}\right)$, where $h=\ell_{1} \ldots \ell_{n}$ (resp. $h=\ell_{2} \ell_{3} \cdots \ell_{n}+\cdots+$ $\left.\ell_{1} \cdots \ell_{j-1} \ell_{j+1} \cdots \ell_{n}+\cdots+\ell_{1} \cdots \ell_{n-1}\right)$.

If $m_{1}=\cdots m_{n}=m$, then the above projective schemes are called respectively $m$-fat lattice and $m$-fat complete grid.

At first we consider fat complete grid of lines in $\mathbb{P}_{K}^{3}$ and we recall the following result of [8].

Theorem 3.2. (see [8]) Let $\mathbb{Y} \subset \mathbb{P}_{K}^{3}$ be an $m$-fat complete grid of type $\left(\ell_{1}, \ell_{2}, \ell_{3}\right)$. Consider the linear forms $L_{j, i}:=x_{j}-a_{j i} x_{0}$ as in Proposition 2.6 and, for $0 \leq q \leq\left[\frac{m}{2}\right]$, the polynomials

$G_{1, q}=L_{1,1}^{q} \cdots L_{1, \ell_{1}}^{q} L_{2,1}^{m-q} \cdots L_{2, \ell_{2}}^{m-q} L_{3,1}^{m-q} \cdots L_{3, \ell_{3}}^{m-q}$,

$G_{2, q}=L_{1,1}^{m-q} \cdots L_{1, \ell_{1}}^{m-q} L_{2,1}^{q} \cdots L_{2, \ell_{2}}^{q} L_{3,1}^{m-q} \cdots L_{3, \ell_{3}}^{m-q}$,

$G_{3, q}=L_{1,1}^{m-q} \cdots L_{1, \ell_{1}}^{m-q} L_{2,1}^{m-q} \cdots L_{2, \ell_{2}}^{m-q} L_{3,1}^{q} \cdots L_{3, \ell_{3}}^{q}$.

If $m$ is odd, then the defining ideal $I_{\mathbb{Y}}$ of $\mathbb{Y}$ is minimally generated by the $\frac{3}{2}(m+1)$ polynomials $G_{1, q}, G_{2, q}, G_{3, q}, 0 \leq q \leq \frac{m-1}{2}$. 
If $m$ is even, then the defining ideal $I_{\mathbb{Y}}$ of $\mathbb{Y}$ is minimally generated by the $\frac{3}{2} m+1$ polynomials $G_{1, q}, G_{2, q}, G_{3, q}, 0 \leq q \leq \frac{m-2}{2}$, and $G:=G_{1, \frac{m}{2}}=G_{2, \frac{m}{2}}=$ $G_{3, \frac{m}{2}}$.

Now we prove that the above ideal $I_{\mathbb{Y}}$ is an 1-lifting of a monomial ideal.

Theorem 3.3. Let $\mathbb{Y} \subset \mathbb{P}_{K}^{3}$ be an $m$-fat complete grid of type $\left(\ell_{1}, \ell_{2}, \ell_{3}\right)$ and consider the linear forms $L_{j, i}=x_{j}-a_{j i} x_{0}$ of Proposition 2.6. Let $\mathbb{A}$ be an 1-lifting matrix of linear forms whose $j$-th row begins with the sequence $L_{j, 1}, L_{j, 2}, \cdots, L_{j, \ell_{j}}$ repeated $\left(1+\left[\frac{m}{2}\right]\right)$-times, $j=1,2,3$.

Then the defining ideal $I_{\mathbb{Y}}$ of $\mathbb{Y}$ is the 1-lifting via the matrix $\mathbb{A}$ of the monomial ideal $\Gamma=\left(x_{1}^{\ell_{1}}, x_{2}^{\ell_{2}}\right)^{m} \cap\left(x_{1}^{\ell_{1}}, x_{3}^{\ell_{3}}\right)^{m} \cap\left(x_{2}^{\ell_{2}}, x_{3}^{\ell_{3}}\right)^{m}$.

Proof. The scheme $\mathbb{Y}$ is supported on the complete grid $Y$ (case $m=1$ ) of type $\left(\ell_{1}, \ell_{2}, \ell_{3}\right)$ whose defining ideal $I_{Y}$ is the 1-lifting of the monomial ideal $J^{\prime}=\left(x_{1}^{\ell_{1}} x_{2}^{\ell_{2}}, x_{1}^{\ell_{1}} x_{3}^{\ell_{3}}, x_{2}^{\ell_{2}} x_{3}^{\ell_{3}}\right)=\left(x_{1}^{\ell_{1}}, x_{2}^{\ell_{2}}\right) \cap\left(x_{1}^{\ell_{1}}, x_{3}^{\ell_{3}}\right) \cap\left(x_{2}^{\ell_{2}}, x_{3}^{\ell_{3}}\right)$ induced by the matrix $\Lambda=\left(L_{j, i}\right)$ of Proposition 2.6.

Now, for $m \geq 2$, we find a minimal system of monomial generators of the ideal $\Gamma$. They are the least common multiples l.c.m. $\left(g_{1}, g_{2}, g_{3}\right)$ with $g_{k}$ minimal monomial generator of

$$
\left(x_{i}^{\ell_{i}}, x_{j}^{\ell_{j}}\right)^{m}=\left(x_{i}^{m \ell_{i}}, x_{i}^{(m-1) \ell_{i}} x_{j}^{\ell_{j}}, x_{i}^{(m-2) \ell_{i}} x_{j}^{2 \ell_{j}}, \ldots, x_{i}^{\ell_{i}} x_{j}^{(m-1) \ell_{j}}, x_{j}^{m \ell_{j}}\right),
$$

$i, j, k \in\{1,2,3\}, k \notin\{i, j\}$.

For $0 \leq q \leq\left[\frac{m}{2}\right]$, put

$\gamma_{1, q}=x_{1}^{q \ell_{1}} x_{2}^{(m-q) \ell_{2}} x_{3}^{(m-q) \ell_{3}}$

$\gamma_{2, q}=x_{1}^{(m-q) \ell_{1}} x_{2}^{q \ell_{2}} x_{3}^{(m-q) \ell_{3}}$

$\gamma_{3, q}=x_{1}^{(m-q) \ell_{1}} x_{2}^{(m-q) \ell_{2}} x_{3}^{q \ell_{3}}$.

If $m$ is odd, then we can prove that $\Gamma \subset K\left[x_{1}, x_{2}, x_{3}\right]$ is generated by $\left\{\gamma_{1, q}, \gamma_{2, q}, \gamma_{3, q}: 0 \leq q \leq \frac{m-1}{2}\right\}$.

If $m$ is even, then $\Gamma \subset K\left[x_{1}, x_{2}, x_{3}\right]$ is generated by $\left\{\gamma_{1, q}, \gamma_{2, q}, \gamma_{3, q}: 0 \leq q \leq \frac{m-2}{2}\right\} \cup\{\gamma\}, \gamma:=\gamma_{1, \frac{m}{2}}=\gamma_{2, \frac{m}{2}}=\gamma_{3, \frac{m}{2}}$.

Thus the defining ideal $I_{\mathbb{Y}}$ of $\mathbb{Y}$ is the 1-lifting of the monomial ideal $\Gamma$ induced by the matrix $\mathbb{A}$.

By means of 1-liftings of monomial ideals, we can describe $m$-fat lattices and $m$-fat complete grids in $\mathbb{P}_{K}^{n}$ also for $n>3$.

Theorem 3.4. Let $\mathbb{X} \subset \mathbb{P}_{K}^{n}$ be an $m$-fat lattice of type $\left(\ell_{1}, \ldots, \ell_{n}\right)$ and consider the linear forms $L_{j, i}=x_{j}-a_{j i} x_{0}$ as in Proposition 2.6 induced by the lattice $X$ of points support of $\mathbb{X}$. Let $\mathbb{A}$ be an 1-lifting matrix of linear forms 
whose $j$-th row begins with the sequence $L_{j, 1}, L_{j, 2}, \cdots, L_{j, \ell_{j}}$ repeated $m$-times, $j=1, \ldots, n$.

Then the defining ideal $I_{\mathbb{X}}$ of $\mathbb{X}$ is the 1-lifting via the matrix $\mathbb{A}$ of the monomial ideal $J^{m}$, where $J=\left(x_{1}^{\ell_{1}}, x_{2}^{\ell_{2}}, \ldots, x_{n}^{\ell_{n}}\right)$ is the monomial ideal giving the lattice $X$.

Proof. The ideal $I_{X}$ defining the lattice $X$ of points is the 1-lifting of $J$ via the matrix $\Lambda=\left(L_{j, i}\right)$ of Proposition 2.6, we have

$$
I_{X}=\cap_{1 \leq i_{k} \leq \ell_{k}, 1 \leq k \leq n}\left(L_{1, i_{1}}, L_{2, i_{2}}, \ldots, L_{n, i_{n}}\right) .
$$

Then the (saturated) ideal defining the $m$-fat lattice $\mathbb{X}$ is

$$
I_{\mathbb{X}}=\cap_{1 \leq i_{k} \leq \ell_{k}, 1 \leq k \leq n}\left(L_{1, i_{1}}, L_{2, i_{2}}, \ldots, L_{n, i_{n}}\right)^{m} .
$$

The above equality gives a minimal primary decomposition of $I_{\mathbb{X}}$. Note that the 1-lifting of $J^{m}$ via the matrix $\mathbb{A}$ is just $I_{X}^{m}=\left(L_{1,1} \cdots L_{1, \ell_{1}}, \ldots, L_{n, 1} \cdots L_{n, \ell_{n}}\right)^{m}$.

We have to prove that $I_{\mathbb{X}}=I_{X}^{m}$.

The lattice $X$ of points is contained in the affine open set $x_{0} \neq 0$. In the localized ring $R_{x_{0}}$ the points of $X$ are defined by coprime maximal ideals $\mathfrak{m}_{k}$, $k=1, \ldots, h$ and we have $I_{X} R_{x_{0}}=\cap_{k=1}^{h} \mathfrak{m}_{k}=\Pi_{k=1}^{h} \mathfrak{m}_{k}$. We have also $I_{\mathbb{X}}$ $R_{x_{0}}=\cap_{k=1}^{h} \mathfrak{m}_{k}^{m}=\Pi_{k=1}^{h} \mathfrak{m}_{k}^{m}=\left(I_{X} R_{x_{0}}\right)^{m}=I_{X}^{m} R_{x_{0}}$. Recall that for an ideal $\mathfrak{a}$ of $R$ a minimal primary decomposition of $\mathfrak{a} R_{x_{0}}$ is given by the primary ideals $\mathfrak{q}$ of a minimal primary decomposition of $\mathfrak{a}$ such that the prime ideal $\sqrt{\mathfrak{q}}$ does not meet $\left\{x_{0}^{i}: i \in \mathbb{N}\right\}$. We could have $I_{X}^{m}=I_{\mathbb{X}} \cap \mathfrak{q}$, with $\sqrt{\mathfrak{q}}=\left(x_{0}, x_{1}, \ldots, x_{n}\right)$, but this case is not possible because the ideal $I_{X}^{m}$, being an 1-lifting of a monomial ideal, is saturated (see [11] Corollary 2.10). So we have $I_{X}^{m}=I_{\mathbb{X}}$.

Theorem 3.5. Let $Y \subset \mathbb{P}_{K}^{n}$ be a complete grid of lines of type $\left(\ell_{1}, \ldots, \ell_{n}\right)$ and $\mathbb{Y} \subseteq \mathbb{P}_{K}^{n}$ be the $m$-fat complete grid supported on $Y$. Consider the linear forms $L_{j, i}=x_{j}-a_{j i} x_{0}$ as in Theorem 2.6 induced by the lattice $X$ of points basis of $Y$ and an 1-lifting matrix $\mathbb{A}$ of linear forms whose $j$-th row begins with the sequence $L_{j, 1}, L_{j, 2}, \cdots, L_{j, \ell_{j}}$ repeated $m$-times, $j=1, \ldots, n$.

Then the defining ideal $I_{\mathbb{Y}}$ of $\mathbb{Y}$ is the 1-lifting via the matrix $\mathbb{A}$ of the monomial ideal $\Gamma=\cap_{k=1}^{n} J_{k}^{m}$, where $J_{k}=\left(x_{1}^{\ell_{1}}, \ldots, \widehat{x_{k}^{\ell_{k}}}, \ldots, x_{n}^{\ell_{n}}\right)$.

Proof. The complete grid $Y$ is the 1-lifting of the monomial ideal $J^{\prime}=$ $\cap_{k=1}^{n} J_{k}$ via the matrix $\Lambda=\left(L_{j, i}\right)$ of Theorem 2.6 and we can write $Y=\cup_{k=1}^{n} Y_{k}$, where $Y_{k}$ denotes the union of the lines contained in $Y$ passing through $P_{\infty, k}$. Note that $Y_{k}$ is defined by the (saturated) ideal 1-lifting via $\Lambda$ of the monomial 
ideal $J_{k}$. Then as in the proof of Theorem 3.4 we obtain that the scheme $\mathbb{Y}_{k}$ of the $m$-fat lines of $Y_{k}$ is just the 1-lifting of the monomial ideal $J_{k}^{m}$ via the matrix $\mathbb{A}$ and the result is obtained.

Corollary 3.6. A m-fat complete grid of lines is A.C.M.

Proof. The $m$-fat complete grids of lines in $\mathbb{P}_{K}^{n}$ are 1-liftings of monomial ideals and then they are A.C.M., by Proposition 1.4.

Remark 3.7. In [8] the statement that the $m$-fat complete grids of lines in the projective space $\mathbb{P}_{K}^{3}$ are A.C.M. was proved by studying the algebraic intersection with a generic plane.

\section{Generalization of Complete Grids in $\mathbb{P}_{K}^{3}$}

In this section we consider an extension (see Definition 4.2) of complete grids in $\mathbb{P}_{K}^{3}$. For configuration of lines we mean a (reduced) finite union of lines. We give a class of configurations of lines that are A.C.M. and that are not constructed by pseudo-1-liftings of monomial ideals. We prove the A.C.M. property by studying the algebraic intersection with hyperplanes as in [7] Theorem 10 for complete grids of lines. We apply the following Lemma

Lemma 4.1. (Lemma 9 of [7]) Let $Z$ be a finite union of $h$ lines in $\mathbb{P}_{K}^{n}$ and $H$ be a linear homogeneous form such that the hyperplane $H=0$ does not contain any line of $Z$ nor any points of $\operatorname{Sing}(Z)$, which are the points intersection of the lines of $Z$. Then $Z$ is A.C.M. if and only if $\left(I_{Z}, H\right)=$ $\cap_{i=1}^{h}\left(\mathcal{P}_{i}, H\right)$, where $\mathcal{P}_{i}$ for $i=1, \ldots, h$ denote the ideals defining the lines of $Z$ and $I_{Z}=\cap_{i=1}^{h} \mathcal{P}_{i}$ is the ideal defining $Z$.

Definition 4.2. In $\mathbb{P}_{K}^{3}$ consider a point $V, s$ distinct lines $m_{1}, \ldots, m_{s}$ passing through $V, h$ planes $\sigma_{1}, \ldots, \sigma_{h}$ do not passing trough $V$ and do not containing the lines $m_{i}, i=1, \ldots, s$. For $i=1, \ldots, s$, denote by $\lambda_{i}$ the plane determined by the edges $m_{i}$ and $m_{i+1}$, putting $m_{s+1}=m_{1}$. Suppose that the planes $\lambda_{1}, \ldots, \lambda_{s}$ are distincts. Denote by $P_{i, j}$ the point intersection of the line $m_{i}$ with the plane $\sigma_{j}$ and suppose that the set $\left\{P_{i, j}: 1 \leq i \leq s, 1 \leq j \leq h\right\}$ consists of sh distinct points. For every $1 \leq i \leq s$ and $1 \leq j \leq h$, denote by $\ell_{i, j}$ the line of the plane $\sigma_{j}$ jointing the points $P_{i, j}$ and $P_{i+1, j}$, putting $P_{s+1, j}=P_{1, j}$.

We call configuration $\boldsymbol{Z}$ the union of the $s(h+1)$ lines $m_{1}, \ldots, m_{s}$ (called edges) and $\left\{\ell_{i, j}: 1 \leq i \leq s, 1 \leq j \leq h\right\}$. The point $V$ is called vertex of $\boldsymbol{Z}$, the planes $\sigma_{1}, \ldots, \sigma_{h}$ are called base-faces of $\boldsymbol{Z}$ and the planes $\lambda_{1}, \ldots, \lambda_{s}$ defined above are called side-faces of $\boldsymbol{Z}$. 
The union of lines $\mathbf{Z}$ can assume various configurations: complete grids in $\mathbb{P}_{K}^{3}$ (see Remark 4.3), tetrahedron (see Example 4.7), parallelepiped (see Example 4.9),pyramid (see Example 4.6 and 4.12), etc... We do not know in general when a configuration of type $\mathbf{Z}$ is a 1-lifting or pseudo-1-lifting. In the following we provide serious examples that show the situation is very complicated, but all three situations can appear: 1-lifting , pseudo-1-lifting, not pseudo-1-lifting.

Remark 4.3. The Definition 4.2 in $\mathbb{P}_{K}^{3}$ generalizes the notion of complete grid. In fact, a complete grid of $\mathbb{P}_{K}^{3}$ can be see as configuration $\mathbf{Z}$ with vertex one of the points at infinity $P_{\infty, 1}, P_{\infty, 2}, P_{\infty, 3}$.

Lemma 4.4. Consider $L_{1}, \ldots, L_{s}, M_{1}, \ldots, M_{h}, H$ linear homogeneous form in

$K\left[x_{0}, x_{1}, x_{2}, x_{3}\right]$ and put $L:=L_{1} \cdots L_{s}$.

1. If $L_{i}, L_{j}, M_{k}$ are linearly independent for every $k$ and $i \neq j$ and $L_{i}, M_{j}, M_{k}$ are linearly independent for every $i$ and $j \neq k$, then $\left(L, M_{1}\right) \cap \cdots \cap$ $\left(L, M_{h}\right)=\left(L, M_{1} \cdots M_{h}\right)$.

2. If $L_{i}, L_{j}, M_{k}, H$ are linearly independent for every $k$ and $i \neq j$ and $L_{i}, M_{j}, M_{k}, H$ are linearly independent for every $i$ and $j \neq k$, then

$$
\left(L, M_{1}, H\right) \cap \cdots \cap\left(L, M_{h}, H\right)=\left(L, M_{1} \cdots M_{h}, H\right) .
$$

Proof. Linearly independent linear forms generate a prime ideal. The proof is a computation based on properties of prime ideals (see [7] Lemma 7)

Proposition 4.5. The configuration $\boldsymbol{Z} \subset \mathbb{P}_{K}^{3}$ described in Definition 4.2 is A.C.M.

Proof. We can suppose $V=(0,0,0,1)$ (so in the open affine set $x_{0} \neq 0$, the lines $m_{1}, \ldots, m_{s}$ are parallel to the third axe $\left.z=\frac{x_{3}}{x_{0}}\right)$. We calculate the ideal $I_{Z} \subset R=K\left[x_{0}, x_{1}, x_{2}, x_{3}\right]$ defining $Z$.

On the plane $x_{3}=0$, whose coordinate ring is $K\left[x_{0}, x_{1}, x_{2}\right]$, consider the points $P_{1}, \ldots, P_{s}$ given by the intersection with the edges $m_{1}, \ldots, m_{s}$ respectively and for every $i=1, \ldots, s$ the line $\ell_{i}$ jointing the points $P_{i}$ and $P_{i+1}$, putting $P_{s+1}=P_{1}$. The line $\ell_{i}$ in $\mathbb{P}^{3}$ can be defined by an ideal $\left(L_{i}, x_{3}\right)$, where $L_{i}$ is a linear homogeneous form of $K\left[x_{0}, x_{1}, x_{2}\right], i=1, \ldots, s$. Note that in $\mathbb{P}^{3}$ the equation $L_{i}=0$ define the side-face $\lambda_{i}$. Let $W$ be the set of points $P_{1}, \ldots, P_{s}$. Note that $W$ in the plane $x_{3}=0$ is defined by the ideal 
$I_{W}=\left(L_{1}, L_{2}\right) \cap\left(L_{2}, L_{3}\right) \cap \cdots \cap\left(L_{s-1}, L_{s}\right) \cap\left(L_{s}, L_{1}\right) \subset K\left[x_{0}, x_{1}, x_{2}\right]$. So the union $Y$ of the edges $m_{1}, \ldots, m_{s}$ in $\mathbb{P}_{K}^{3}$ is defined by the ideal $I_{Y}=I_{W} K\left[x_{0}, x_{1}, x_{2}, x_{3}\right]$.

If the base-face $\sigma_{j} \subset \mathbb{P}_{K}^{3}$ is defined by a linear homogeneous form $M_{j}$, then the edge $\ell_{i, j} \subset \mathbb{P}_{K}^{3}$ is defined by the ideal $\left(L_{i}, M_{j}\right) \subset K\left[x_{0}, x_{1}, x_{2}, x_{3}\right], 1 \leq j \leq h$, $1 \leq i \leq s$.

The ideal $I_{Z}$ defining $Z$ is :

$I_{Y} \cap\left(L_{1}, M_{1}\right) \cap \cdots \cap\left(L_{s}, M_{1}\right) \cap\left(L_{1}, M_{2}\right) \cap \cdots \cap\left(L_{s}, M_{2}\right) \cap \cdots \cap\left(L_{1}, M_{h}\right) \cap \cdots \cap\left(L_{s}, M_{h}\right)$.

For $i \neq j$ the linear forms $L_{i}, L_{j}, M_{k} \in K\left[x_{0}, x_{1}, x_{2}, x_{3}\right]$ are linearly independent and for $j \neq k$ the linear forms $L_{i}, M_{j}, M_{k} \in K\left[x_{0}, x_{1}, x_{2}, x_{3}\right]$ are linearly independent, so by Lemma 4.4 we have $I_{Z}=$ $I_{Y} \cap\left(L_{1} L_{2} \cdots L_{s}, M_{1}\right) \cap\left(L_{1} L_{2} \cdots L_{s}, M_{2}\right) \cap \cdots \cap\left(L_{1} L_{2} \cdots L_{s}, M_{h}\right)=$ $I_{Y} \cap\left(L_{1} L_{2} \cdots L_{s}, M_{1} M_{2} \cdots M_{h}\right)$.

Since $L_{1} L_{2} \cdots L_{s} \in I_{Y}$, by the modular low we have

$$
I_{Z}=\left(L_{1} L_{2} \cdots L_{s}\right)+\left(\left(M_{1} M_{2} \cdots M_{h}\right) \cap I_{Y}\right) .
$$

Put $I_{W}=\left(f_{1}, \ldots, f_{r}\right) \subset K\left[x_{0}, x_{1}, x_{2}\right]$, since $I_{W} K\left[x_{0}, x_{1}, x_{2}, x_{3}\right]=I_{Y}$ is intersection of prime ideals do not containing the linear form $M_{j}$, for every $j=1, \ldots, h$ (i.e. every line $m_{i}$ is not contained in the plane $\sigma_{j}$ ), we obtain

$$
I_{Z}=\left(L_{1} L_{2} \cdots L_{s}, M_{1} M_{2} \cdots M_{h} f_{1}, \ldots, M_{1} M_{2} \cdots M_{h} f_{r}\right) .
$$

Now we consider a linear homogeneous form $H \in K\left[x_{0}, x_{1}, x_{2}, x_{3}\right]$ satisfying conditions of Lemma 4.1. The configuration $Y$ of lines is A.C.M., in fact its coordinate ring $\left(K\left[x_{0}, x_{1}, x_{2}\right] / I_{W}\right)\left[x_{3}\right]$ is C.M. since $W$ is a finite set of distinct points. Thus $\left(I_{Y}, H\right)=\left(L_{1}, L_{2}, H\right) \cap \cdots \cap\left(L_{s}, L_{1}, H\right)$.

Note that for $i \neq j$ the linear forms $L_{i}, L_{j}, M_{k}, H$ are linearly independent and for $j \neq k$ the linear forms $L_{i}, M_{j}, M_{k}, H$ are linearly independent, so by Lemma 4.4 we have $\left(L_{1}, M_{1}, H\right) \cap \cdots \cap\left(L_{s}, M_{h}, H\right)=\left(L_{1} \cdots L_{s}, M_{1} \cdots M_{h}, H\right)$.

Since $\left(L_{1} \cdots L_{s}, H\right) \subset\left(I_{Y}, H\right)$, by the modular low we obtain $\left(I_{Y}, H\right) \cap\left(L_{1} \cdots L_{s}, M_{1} \cdots M_{h}, H\right)=\left(L_{1} \cdots L_{s}, H\right)+\left(\left(M_{1} \cdots M_{h}\right) \cap\left(I_{Y}, H\right)\right)$.

Recall that $Y$ is A.C.M., then $\left(I_{Y}, H\right)=\left(f_{1}, \ldots, f_{r}, H\right)$ is the intersection of the prime ideals defining the points intersection of $Y$ with the plane $H=0$. None of those points belongs to the planes $\sigma_{1}, \ldots, \sigma_{h}$. Thus the above ideal is $\left(L_{1} \cdots L_{s}, H, M_{1} \cdots M_{h} f_{1}, \ldots, M_{1} \cdots M_{h} f_{r}, M_{1} \cdots M_{h} H\right)=\left(I_{Z}, H\right)$. Then $Z$ is A.C.M.

Now we describe some figures of the euclidean space $\mathbb{E}_{\mathbb{R}}^{3}$ by using configurations of lines in the real projective space $\mathbb{P}_{\mathbb{R}}^{3}$ forming a configuration $\mathbf{Z}$ (Definition 4.2). They generalize examples described in [6]. We see when they are 
related to pseudo-1-liftings of monomial ideals of $K\left[x_{1}, x_{2}, x_{3}\right]$. The coordinates of $\mathbb{E}_{\mathbb{R}}^{3}$ are denoted as usual $x=\frac{x_{1}}{x_{0}}, y=\frac{x_{2}}{x_{0}}, z=\frac{x_{3}}{x_{0}}$.

Example 4.6. Pyramid. A configuration defined by a pseudo-1-lifting of the monomial ideal giving the complete grids of lines of type $(2,2,1)$. Consider the pyramid with vertices $P_{1}=(0,0,0), \quad P_{2}=(1,0,0), P_{3}=(0,1,0), P_{4}=$ $(1,1,0), P_{5}=(0,0,1)$, whose faces are the planes $x=0, y=0, x+z-1=0$, $y+z-1=0$ and $z=0$ (Figure 8 ). The projective closure of the configuration of the 8 edges of the pyramid is given by the pseudo-1-lifting of the monomial ideal $J=\left(x_{1}^{2} x_{2}^{2}, x_{1}^{2} x_{3}, x_{2}^{2} x_{3}\right)$ (see Figure 9) induced by the matrix $A$, but is not a 1-lifting of $J$ because the linear forms $L_{j, i}$ of $A$ do not belong to $K\left[x_{0}, x_{j}\right] \forall j=$ $1,2,3, \quad \forall i=1,2$ :

$$
A=\left(\begin{array}{cc}
x_{1} & x_{1}+x_{3}-x_{0} \\
x_{2} & x_{2}+x_{3}-x_{0} \\
x_{3} & L_{3,2}
\end{array}\right)
$$

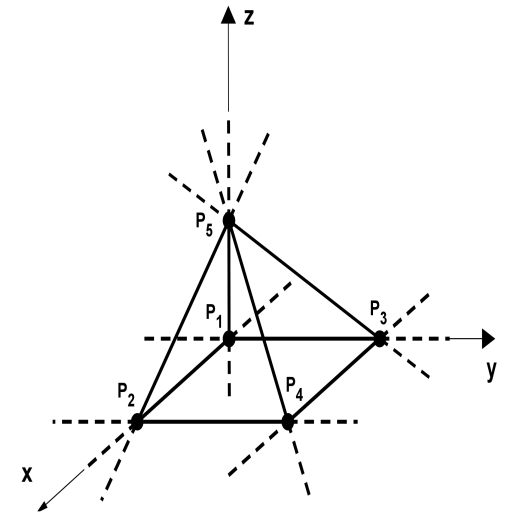

Figure 8: The pyramid. Its projective closure is a pseudo-1-lifting of a monomial ideal considered in Def. 2.8 .

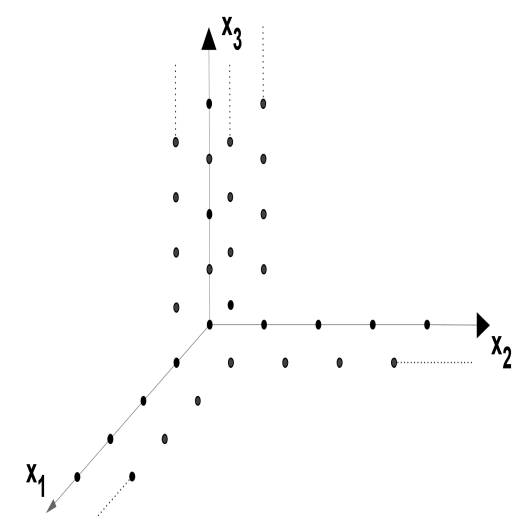

Figure 9: The order ideal in $\mathbb{N}^{3}$ of $J=\left(x_{1}^{2} x_{2}^{2}, x_{1}^{2} x_{3}, x_{2}^{2} x_{3}\right)$ giving the pyramid.

To obtain a regular pyramid take the vertices $P_{1}, P_{2}, P_{3}, P_{4}$ as above and $P_{5}^{\prime}=\left(\frac{1}{2}, \frac{1}{2}, \frac{\sqrt{2}}{2}\right)$. Its projective closure in $\mathbb{P}_{\mathbb{R}}^{3}$ is the pseudo-1-lifting of the monomial ideal $J$ induced by the matrix

$$
A^{\prime}=\left(\begin{array}{cc}
\sqrt{2} x_{1}-x_{3} & \sqrt{2} x_{1}+x_{3}-\sqrt{2} x_{0} \\
\sqrt{2} x_{2}-x_{3} & \sqrt{2} x_{2}+x_{3}-\sqrt{2} x_{0} \\
x_{3} & L_{3,2}
\end{array}\right) .
$$


Example 4.7. Tetrahedron. It is defined by a pseudo-1-lifting of a monomial ideal not giving complete grids of lines. Consider a tetrahedron with vertices $P_{1}=(0,0,0), \quad P_{2}=(1,0,0), \quad P_{3}=(0,1,0), P_{4}=(0,0,1)$, whose faces are the planes $x=0, \quad y=0, \quad x+y+z-1=0$ and $z=0$. Let $Z$ be the configuration of the 6 edges of the tetrahedron (Figure 10). Then projective closure $\bar{Z}$ of $Z$ is given by the pseudo-1-lifting $I$ of the monomial ideal $J=\left(x_{1}^{2} x_{2}, x_{1}^{2} x_{3}, x_{2}^{2} x_{3}, x_{1} x_{2} x_{3}\right)$ (see Figure 11) induced by the matrix $B$, but is not a 1-lifting of $J$ because the linear forms $L_{j, i}$ of $B$ do not belong to $K\left[x_{0}, x_{j}\right] \forall j=1,2,3, \quad \forall i=1,2$ :

$$
B=\left(\begin{array}{cc}
x_{1} & x_{1}+x_{2}+x_{3}-x_{0} \\
x_{2} & x_{2}+x_{3}-x_{0} \\
x_{3} & L_{3,2}
\end{array}\right)
$$

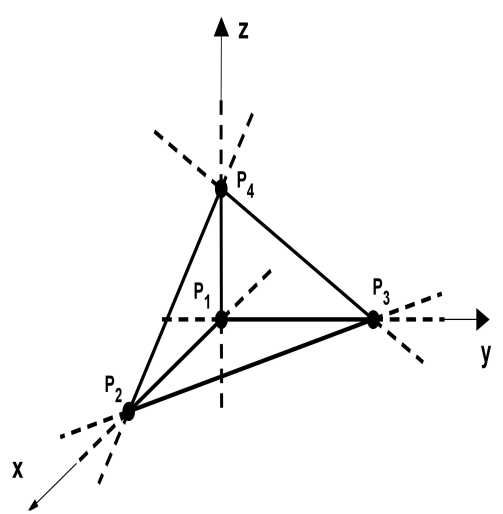

Figure 10: The tetrahedron. Its projective closure is a pseudo-1lifting of a monomial ideal not satisfying Def. 2.8.

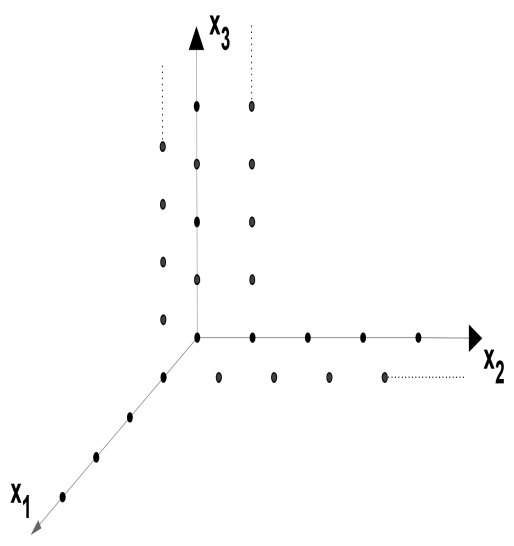

Figure 11: The order ideal in $\mathbb{N}^{3}$ of $J=\left(x_{1}^{2} x_{2}, x_{1}^{2} x_{3}, x_{2}^{2} x_{3}, x_{1} x_{2} x_{3}\right)$ giving the tetrahedron.

Note that the above matrix does not satisfy Condition $\left(\alpha^{\prime}\right)$, however the pseudo-1-lifting $I$ define a reduced scheme. For the linear forms $L_{j, i}$ entries of $B$ we have that the vector spaces $\left\langle L_{1,1}, L_{2,2}, L_{3,1}>\right.$ and $\left\langle L_{1,2}, L_{2,2}, L_{3,1}>\right.$ are equal, but the plane $L_{2,2}=0$ is not a face of the tetrahedron $\bar{Z}$ defined by $I$.

Remark 4.8. If we consider the pseudo-1-lifting of the above monomial 
ideal $J$ via the matrix

$$
C=\left(\begin{array}{cc}
x_{1} & x_{1}+\frac{1}{2} x_{2}+x_{3}-x_{0} \\
x_{2} & x_{2}+x_{3}-x_{0} \\
x_{3} & L_{3,2}
\end{array}\right)
$$

satisfying Condition $\left(\alpha^{\prime}\right)$, we obtain a configuration $Y$ of 6 lines that is neither a tetrahedron nor a configuration satisfying Theorem 3 of [6].

Example 4.9. Parallelepiped. Configuration defined by a pseudo-1lifting of the monomial ideal giving the complete grids of lines of type $(2,2,2)$. Consider in $\mathbb{E}_{\mathbb{R}}^{3}$ a solid with vertices $P_{i}=\left(a_{i}, b_{i}, 0\right), Q_{i}=\left(a_{i}, b_{i}, 1\right), i=$ $1,2,3,4$ (see Figure 12). The projective closure $Z \subset \mathbb{P}_{\mathbb{R}}^{3}$ of the 12 egdes of the solid is a configuration $\mathbf{Z}$ of Definition 4.2 with two quadrilaterals as bases and with vertex the point $V=(0,0,0,1) \in \mathbb{P}_{\mathbb{R}}^{3}$. $Z$ is defined by the the pseudo1-lifting of the monomial ideal $J=\left(x_{1}^{2} x_{2}^{2}, x_{1}^{2} x_{3}^{2}, x_{2}^{2} x_{3}^{2}\right)$ (see Figure 13) induced by the matrix

$$
M=\left(\begin{array}{cc}
\Lambda_{1} & \Lambda_{3} \\
\Lambda_{2} & \Lambda_{4} \\
x_{3} & x_{3}-x_{0}
\end{array}\right)
$$

where, for $1 \leq i \leq 4, \Lambda_{i}=\left(b_{i+1}-b_{i}\right)\left(x_{1}-a_{i} x_{0}\right)-\left(a_{i+1}-a_{i}\right)\left(x_{2}-b_{i}\right)$, with $a_{5}=a_{1}, b_{5}=b_{1}$. Note that $\Lambda_{i}=0, i=1,2,3,4$, give the 4 side-faces of the solid, while $x_{3}=0, x_{3}-x_{0}=0$ give the base-faces of the solid. The set of the 8 vertices of the solid $\left\{P_{1}, \ldots, P_{4}, Q_{1}, \ldots, Q_{4}\right\}$ are given by the pseudo-1-lifting of $\left(x_{1}^{2}, x_{2}^{2}, x_{3}^{2}\right)$ induced by the above matrix $M$.

To obtain a parallelepiped in $\mathbb{E}_{\mathbb{R}}^{3}$ we can consider a pseudo-1-matrix

$$
M^{\prime}=\left(\begin{array}{cc}
a x_{1}+b x_{2} & a x_{1}+b x_{2}+c x_{0} \\
b x_{1}-a x_{2} & b x_{1}-a x_{2}+d x_{0} \\
x_{3} & x_{3}-x_{0}
\end{array}\right)
$$

Note that for $b=0$ we have that $A^{\prime}$ is an 1-lifting matrix and we obtain a parallelepiped whose projective closure is a complete grid of lines of type $(2,2,2)$.

Example 4.10. Configurations of lines with a regular exagon as bases. The configuration cannot be defined by pseudo-1-liftings of monomial ideals. Consider in $\mathbb{E}_{\mathbb{R}}^{3}$ the solid with a regular exagon as bases, whose vertices are $P_{1}=(0,0,0), P_{2}=\left(\frac{1}{2}, \frac{\sqrt{3}}{2}, 0\right), \quad P_{3}=\left(\frac{3}{2}, \frac{\sqrt{3}}{2}, 0\right), \quad P_{4}=(2,0,0), \quad P_{5}=$ 


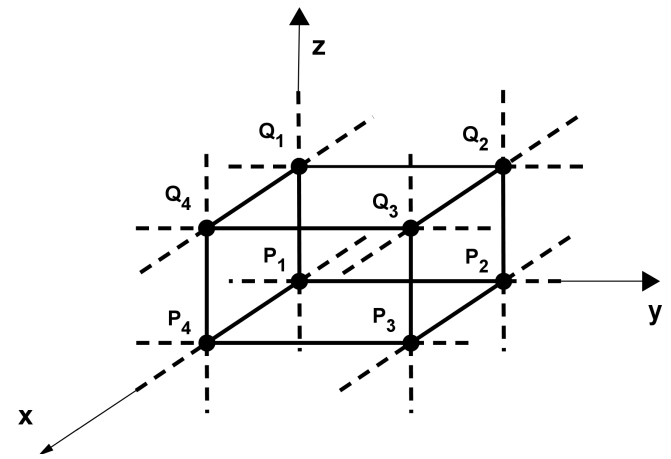

Figure 12: The parallelepiped with rectangular bases. Its projective closure is a complete grid of type $(2,2,2)$ and an 1-lifting of a monomial ideal.

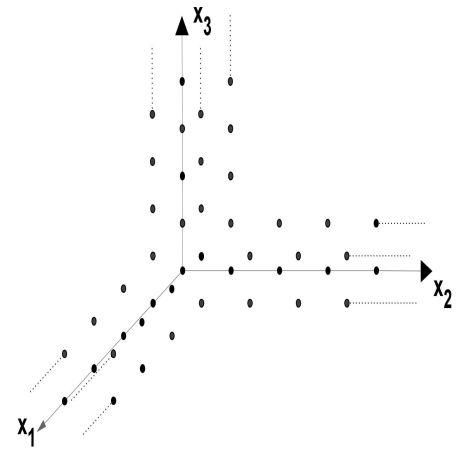

Figure 13: The order ideal in $\mathbb{N}^{3}$ of $J=\left(x_{1}^{2} x_{2}^{2}, x_{1}^{2} x_{3}^{2}, x_{2}^{2} x_{3}^{2}\right)$ giving the parallelepiped.

$\left(\frac{3}{2},-\frac{\sqrt{3}}{2}, 0\right), \quad P_{6}=\left(\frac{1}{2},-\frac{\sqrt{3}}{2}, 0\right)$ and $Q_{1}=(0,0,1), \quad Q_{2}=\left(\frac{1}{2}, \frac{\sqrt{3}}{2}, 1\right), \quad Q_{3}=$ $\left(\frac{3}{2}, \frac{\sqrt{3}}{2}, 1\right), \quad Q_{4}=(2,0,1), \quad Q_{5}=\left(\frac{3}{2},-\frac{\sqrt{3}}{2}, 1\right), \quad Q_{6}=\left(\frac{1}{2},-\frac{\sqrt{3}}{2}, 1\right)$ (Figure 14).

The 8 faces of the solid are the planes $z=0, z-1=0$ and the 6 planes $L_{i}=0$ passing through the vertices $P_{i}, P_{i+1}, Q_{i}, Q_{i+1}$, for $1 \leq i \leq 6$, with $P_{7}=P_{1}$ and $Q_{7}=Q_{1}$. We can calculate $L_{1}=\sqrt{3} x-y, L_{2}=y-\frac{\sqrt{3}}{2}, \quad L_{3}=\sqrt{3} x+y-2 \sqrt{3}$, $L_{4}=\sqrt{3} x-y-2 \sqrt{3}, \quad L_{5}=y+\frac{\sqrt{3}}{2}, \quad L_{6}=\sqrt{3} x+y$. The configuration $Z$ of the edges of that solid consists of 18 lines, its projective closure $\bar{Z}$ is a configuration $\mathbf{Z}$ of Definition 4.2 defined by the ideal $I=\left(\bar{L}_{1} \bar{L}_{2} \cdots \bar{L}_{6}, f_{1} x_{3}\left(x_{3}-x_{0}\right), f_{2} x_{3}\left(x_{3}-\right.\right.$ $\left.x_{0}\right)$ ), where $\bar{L}_{i}=0$ is the projective closure of the plane $L_{i}=0$, for $1 \leq i \leq 6$, $f_{1}=\left(x_{1}-x_{0}\right)^{2}+x_{2}^{2}-x_{0}^{2}$ and $f_{2}=\bar{L}_{1} \bar{L}_{3} \bar{L}_{5}$ (see proof of Proposition 4.5). Then $\bar{Z}$ is contained in a unique minimal surface of degree 4 that cannot be defined by the product of linear forms, so the ideal $I$ is not generated by products of linear forms and it cannot be a pseudo-1-lifting of monomial ideals.

Remark 4.11. Consider the configuration $Z$ of the above Example and the monomial ideal $J=\left(x_{1}^{3} x_{2}^{3}, x_{1}^{3} x_{3}^{2}, x_{2}^{3} x_{3}^{2}\right)$ giving the complete grids of type $(3,3,2)$ (see Figure 15) and the matrix

$$
\Lambda=\left(\begin{array}{ccc}
\bar{L}_{1} & \bar{L}_{3} & \bar{L}_{5} \\
\bar{L}_{2} & \bar{L}_{4} & \bar{L}_{6} \\
x_{3} & x_{3}-x_{0} & L_{3,3}
\end{array}\right)
$$




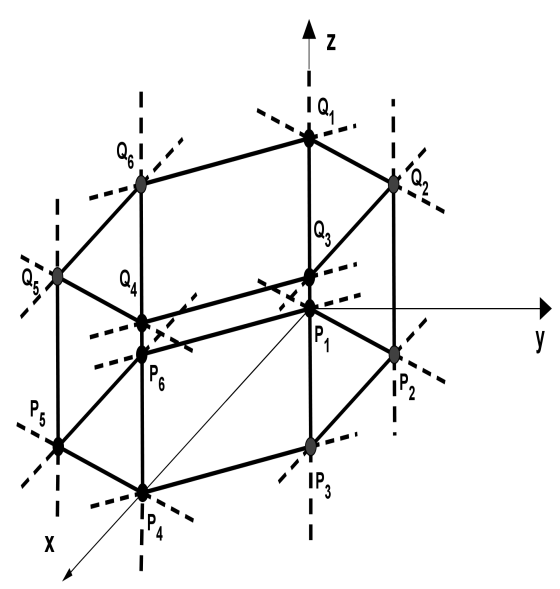

Figure 14: Solid with regular exagon as base. It is a configuration of 18 lines whose projective closure is not a pseudo-1-liftings of monomial ideals.

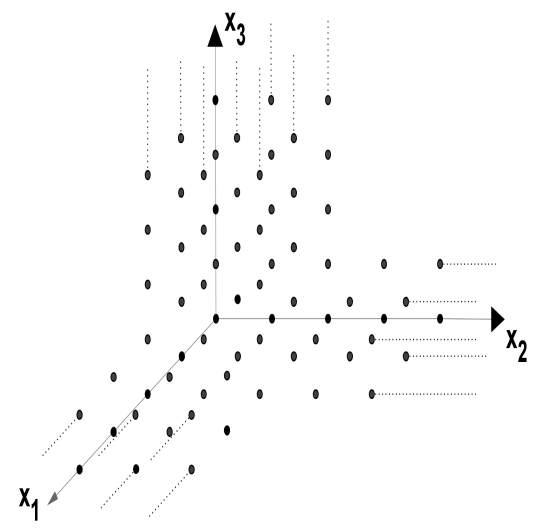

Figure 15: The order ideal in $\mathbb{N}^{3}$ of $J=\left(x_{1}^{3} x_{2}^{3}, x_{1}^{3} x_{3}^{2}, x_{2}^{3} x_{3}^{2}\right)$, giving by means of a pseudo-1-lifting a configuration $Y \subset \mathbb{P}_{\mathbb{R}}^{3}$ of 21 lines such that its intersection with the open set $x_{0} \neq 0$ is the above solid with exagon as base.

The configuration $Y \subset \mathbb{P}_{\mathbb{R}}^{3}$ given by the pseudo-1-lifting of $J$ induced by the matrix $\Lambda$ consists of 21 lines and in the open set $x_{0} \neq 0$ the trace of $Y$ is the set $Z$ of the 18 edges of the solid described in the above Example. On the other hand the projective closure $\bar{Z}$ of $Z$ is obtained from $Y$ by removing the lines defined by the ideals $\left(\bar{L}_{1}, \bar{L}_{4}\right),\left(\bar{L}_{3}, \bar{L}_{6}\right),\left(\bar{L}_{5}, \bar{L}_{2}\right)$.

\section{Example 4.12. Lines of a pyramid with base a regular polygon of} $n \geq 5$ sides. This configuration of lines cannot be defined by pseudo-1-liftings of monomial ideals. Consider in $\mathbb{E}_{\mathbb{R}}^{3}$ the pyramid with base a regular polygon with $n \geq 5$ sides, of vertices $P_{1,1}, \ldots, P_{n, 1}$. The $n$ side-faces $\lambda_{1}, \ldots, \lambda_{n}$ of this pyramid are defined respectively by the linear homogeneous forms $L_{1}, \ldots, L_{n}$, and the only base-face by the linear homogeneous form $M$. The configuration $Z$ of the edges of that pyramid consists of $2 n$ lines, its projective closure $\bar{Z}$ is a configuration $\mathbf{Z}$ of Definition 4.2 defined by the ideal $I=\left(\bar{L}_{1} \bar{L}_{2} \cdots \bar{L}_{n}, f_{1} \bar{M}, \ldots, f_{r} \bar{M}\right)$, where $\bar{L}_{i}=0$ is the projective closure of the plane $L_{i}=0$, for $1 \leq i \leq n$, and $\bar{M}=0$ is the projective closure of the plane $M=0$. Moreover, $f_{1}, \ldots, f_{r}$ are the generators of the ideal $I_{Y}=\left(\bar{L}_{1}, \bar{L}_{2}\right) \cap \ldots \cap\left(\bar{L}_{n-1}, \bar{L}_{n}\right)$ (see proof of Proposition $4.5)$, but $I_{Y}=I_{W} K\left[x_{0}, \ldots, x_{3}\right]$ where $W$ is the set of points $P_{1,1}, \ldots, P_{n, 1}$ then $I_{Y}$ contains a generator of degree 2 because $P_{1,1}, \ldots, P_{n, 1}$ belong to a circumfer- 
ence. Then $\bar{Z}$ is contained in a unique minimal surface of degree 3 that cannot be defined by the product of linear forms, so the ideal $I$ is not generated by products of linear forms and it cannot be a pseudo-1-lifting of monomial ideals.

Finally, we give an example of configuration of lines $Y$ Cohen-Macaulay whose defining ideal $I_{Y}$ is generated by products of linear forms but is not a pseudo-1-lifting nor a lifting of a monomial ideal. The following configuration $Y$ is obtained from a configuration $\mathbf{Z}$ (see Definition 4.2) consisting of the lines: $m_{1}, m_{2}, m_{3}, \ell_{1,1}, \ell_{2,1}, \ell_{3,1}$ (where $\left.P_{1,1}=P_{1}, P_{2,1}=P_{2}, P_{3,1}=P_{3}, V=P_{4}\right)$ and eliminating the lines $m_{2}, \ell_{3,1}$.

Example 4.13. Consider in $\mathbb{P}_{\mathbb{R}}^{3}$ the following points: $P_{1}=(7,1,5,-2), P_{2}=$ $(3,5,1$,

$-2), P_{3}=(7,1,-3,-2), P_{4}=(3,-3,1,-2)$ and we construct the lines through the points $P_{i}$ and $P_{i+1},(i=1,2,3,4)$, where $P_{5}=P_{1}$. The defining ideal $I_{Y}$ the configuration $Y$ of these four lines is $I_{Y}=((x-z+t)(x+z+2 t),(x-$ $y+3 t)(x+y+4 t))$, hence $I_{Y}$ is generated by products of linear forms. Moreover since $I_{Y}$ is a complete intersection it is A.C.M. But, $I_{Y}$ is not a pseudo1-lifting of a monomial ideal $J$, otherwise $J$ must be unmixed of dimension 1 , the order ideal $\mathcal{N}(J)$ must consist of four lines: the three axes and a line parallel to an axis, hence a less of a change of coordinates we have that $J=(x, y) \cap(y, z) \cap\left(x^{2}, z\right)=\left(x z, y z, x^{2} y\right)$, then $J$ has a minimal generator of degree 3 (while $I_{Y}$ is generated in degree 2). But $I_{Y}$ and $J$ must have the same minimal resolution, contradiction.

\section{Acknowledgments}

Authors were supported by MIUR and GNSAGA (CNR).

\section{References}

[1] A. BJörner, I. Peeva, J. Sidman, Subspace arrangements defined by products of linear forms, J. London Math. Soc., 71, No.2 (2005), 273-288, doi: 10.1112/S0024610705006356.

[2] F. Cioffi, M.G. Marinari, L. Ramella, Geometric description of lifting monomial ideals, Comm. Algebra, 33, No.5 (2005), 1483-1499, doi: 10.1081/AGB-200060519. 
[3] E. Eisenbud, Commutative Algebra with a view towards algebraic geometry, GTM 150, Springer-Verlag, (1995).

[4] Geramita, A.V.; Harima, T.; Shin, Y.S. Decompositions of the Hilbert function of a set of points in $\mathbb{P}^{n}$. Canad. J. Math., 53,(2001), 923-943, doi: 10.4153/CJM-2001-037-3.

[5] M. Guida, Syzygies of grids of projective lines, Ricerche di Matematica, 57, No.1 (2008), 159-167, doi: 10.1007/s11587-008-0027-8.

[6] M. Guida, Ideals of some configurations of lines in $\mathbb{P}_{K}^{n}$ : generators and macaulayness, Ricerche di Matematica, 58, No.2 (2009), 243-247, doi: 10.1007/s11587-009-0060-2.

[7] M. Guida, F. Orecchia, Algebraic properties of grids of projective lines, J. Pure and Appl. Algebra, 208, No.2 (2007), 603-615, doi: 10.1016/j.jpaa.2006.03.015.

[8] M. Guida, F. Orecchia, Algebraic properties of grids of fat lines, Int. J. of Pure and Appl. Math., 40, No.4 (2007), 519-542.

[9] Guerrieri, Anna and Swanson, Irena, On the ideal of minors of matrices of linear forms, Contemp. Math., 331,(2003), 139-152, doi: 10.1090/conm/331/05907.

[10] M.G. Marinari, L. Ramella, Criteria for stability and Borelness, in Zerodimensional schemes and applications (Naples, 2000), Queen's Papers in Pure and Appl. Math., Queen's Univ., Kingston, ON,, 123,(2002), 201217.

[11] J. Migliore, U. Nagel, Lifting monomial ideals, Comm. Algebra, 28, No.12 (2000), 5679-5701, doi: 10.1080/00927870008827182.

[12] Li, Wen Ch'ing Winnie and Li, Shuo-Yen Robert, On generators of ideals associated with unions of linear varieties. Bull. London Math. Soc., 13, No. 1 (1981), 59-65, doi: 10.1112/blms/13.1.59. 